\title{
CROC: Convex Resolution Of Centroidal dynamics trajectories to provide a feasibility criterion for the multi contact planning problem
}

\author{
Pierre Fernbach $^{1}{ }^{2}$, Steve Tonneau ${ }^{1}{ }^{2}$ and Michel Taïx ${ }^{12}$
}

\begin{abstract}
We tackle the transition feasibility problem, that is the issue of determining whether there exists a feasible motion connecting two configurations of a legged robot. To achieve this we introduce CROC, a novel method for computing centroidal dynamics trajectories in multi-contact planning contexts. Our approach is based on a conservative and convex reformulation of the problem, where we represent the center of mass trajectory as a Bezier curve comprising a single free control point as a variable. Under this formulation, the transition problem is solved efficiently with a Linear Program (LP) of low dimension.

We use this LP as a feasibility criterion, incorporated in a sampling-based contact planner, to discard efficiently unfeasible contact plans. We are thus able to produce robust contact sequences, likely to define feasible motion synthesis problems. We illustrate this application on various multi-contact scenarios featuring HRP2 and HyQ.

We also show that we can use CROC to compute valuable initial guesses, used to warm-start non-linear solvers for motion generation methods. This method could also be used for the 0 and 1-Step capturability problem. The source code of CROC is available under an open source BSD-2 License.
\end{abstract}

\section{INTRODUCTION}

Multi-contact motion planning is the problem of automatically computing a feasible motion for a legged robot, from an initial to a goal position, in an arbitrary environment. This problem is one of the main issues preventing the safe deployment of legged robots in environments they never encountered before.

While gaited legged locomotion is commonly achieved on flat surfaces [1], addressing multi-contact locomotion in the general case remains open. A first reason comes from the difficulty of handling non-gaited behaviours. The choice of the contacts to create or break during the motion (which effectors, which locations, and for how long) introduces a combinatorial problem [2]. Moreover, multi-contact motion is not limited to flat and / or coplanar surfaces. Thus simplified dynamic models such as the linear inverted pendulum [1], [3] do not apply. As a result non-convex dynamic constraints must be handled [4], without guarantees of success given modern numerical resolution schemes. The close proximity of obstacles introduces another source of non-convexity, and as a result planning a motion in a complex environment requires a global planning method to avoid getting stuck in a local minima [5].

In this paper we focus on the planning aspect of multi contact motion, that is the issue of finding the contacts that must be created between the effectors and the environment. Finding suitable contact candidates requires checking for

\footnotetext{
${ }^{1}$ CNRS, LAAS, 7 avenue du colonel Roche, F31400 Toulouse, France

${ }^{2}$ Univ de Toulouse, LAAS, F31400 Toulouse, France
}

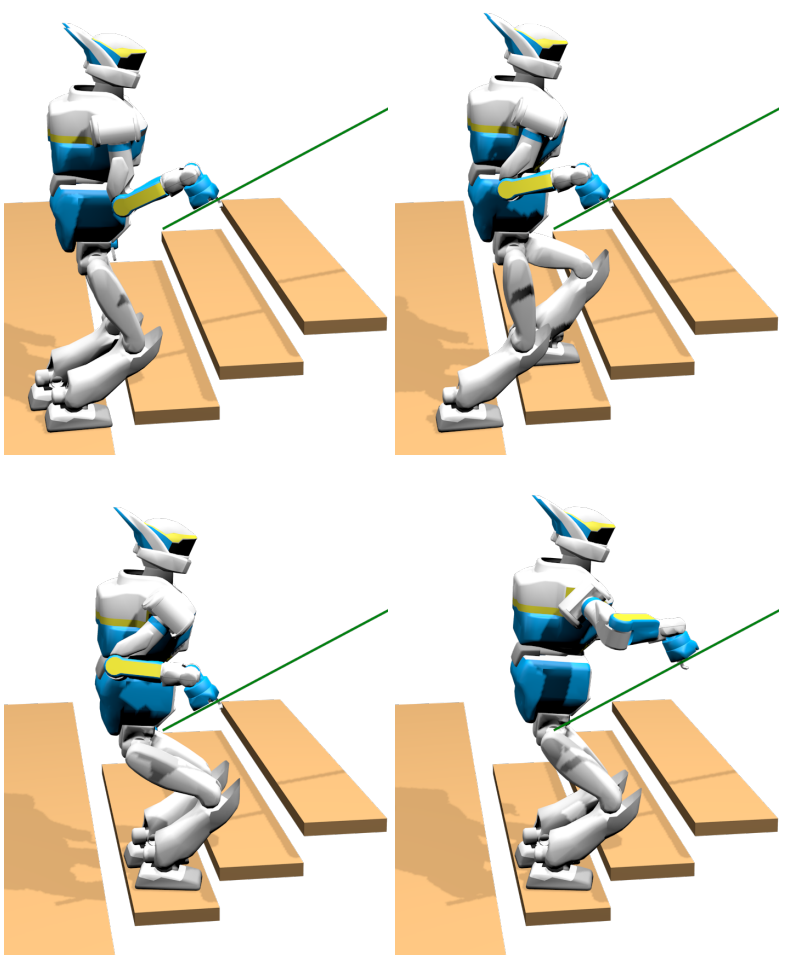

Fig. 1: A feasible multi-contact sequence for a stair climbing motion on the HRP2 robot automatically computed with our contact planner and CROC. At most one contact creation and removal separates two consecutive contact phases. CROC guarantees that between each configuration in the sequence, there exists a feasible centroidal trajectory for the Center Of Mass (including the flying effector phases).

transition feasibility: two consecutive contact states in a plan must be connected by a kinematically and dynamically feasible motion (Figure 1). Significant contributions [6], [2], [7] set the theoretical foundations of the problem for the quasi-static case (where the acceleration remains close to zero), but did not provide a computationally efficient way of addressing it. For these reasons, recent contributions make the assumption that a feasible contact sequence is a given of the problem, and focus on the (already hard) generation of a feasible motion along it [8], [9], [10]. Because of the combinatorial aspect of contact planning, the computational time required by these methods is too important to use a trial-and-error approach to verify the feasibility. Caron et al. recently proposed a computationally efficient method [11], 
but its application range is restricted to single-contact to single-contact transitions. The use of mixed-integer programming allows to compute the contact sequence, though a major issue is that the dynamic feasibility of the contact sequence is not verified before trying to generate the motion [12], which necessarily results in unfeasible problems. This issue was later addressed in [13] through a convex approximation of the dynamic constraints, though the approach remains subject to combinatorial explosion. One noticeable exception comes from the work of Mordatch et al. where the contact locations and the trajectory are simultaneously computed using a simplified model, although performances are not interactive $^{1}$ [14].

In our previous works, we propose heuristics to increase the odds that a computed contact sequence defines a feasible motion generation problem [15], [16], but do not provide strong guarantees of feasibility. More recently we proposed a formal verification of the transition feasibility, but only under the conservative quasi-static case [17].

In this paper, we propose a step towards formal guarantees of transition feasibility in the general case. To prove the feasibility of a contact transition, we introduce CROC, a convex formulation of the transition feasibility problem, efficiently solved as a 3-dimensional Linear Program (LP). Regarding the centroidal dynamics constraints of the motion, CROC is conservative (though not restricted to the quasistatic case), thus the resolution of the problem guarantees the dynamic feasibility. Furthermore, we take into account the kinematic constraints with the inclusion of a necessary condition on the kinematic feasibility.

Our contribution and main novelty is a convex reformulation of the transition feasibility problem, conservative but exact, when convexity is traditionally obtained through a relaxation of the dynamic constraints or in the quasi-static case. CROC has several important additional applications: it can be used for the 0 and 1 step capturability problem [18] [19] [20], and also to compute a valuable initial guess for non-linear motion generation algorithms [8].

After recalling the formal definition of the problem in Section II, we present our approach in Section III. We then demonstrate two applications in Section IV: we first integrate CROC as a filter to improve the robustness of a contact planner [16]; we then use CROC to compute a warm start for a non-linear motion generation algorithms [8], and we show that this results in a computational gain in the convergence of the solver. We present quantitative results showing the interest of these two applications in Section IV, and discuss the limitations and other applications of CROC in Section V.

\section{Problem DEFinition}

In this work we define the transition feasibility problem with respect to the centroidal dynamics of a robot, as now commonly done [21], [9], [8].

\footnotetext{
${ }^{1}$ Several minutes of computation are required to generate a centroidal trajectory and the contact locations along few steps.
}

\section{A. Legged motion is characterized by contact phases}

A contact phase is defined such that: the number and locations of the active contacts remain constant at each phase; each contact phase differs from the previous one by exactly one contact creation or removal. Each contact phase constrains dynamically and kinematically the motion of the robot, and at the transition instant between two phases, the constraints of both phases apply. In Figure 1, between the top left and top right configuration, there is thus three contact phases, including one where the left foot is not in contact.

A state $\mathbf{x}^{\{p\}}=\left(\mathbf{c}^{\{p\}}, \dot{\mathbf{c}}^{\{p\}}, \ddot{\mathbf{c}}^{\{p\}}\right) \in \mathbb{R}^{3} \times \mathbb{R}^{3} \times \mathbb{R}^{3}$ describes a Center Of Mass (COM) position, velocity and acceleration satisfying the constraints at contact phase $\{p\}$. Given two states $\mathbf{x}_{s}^{\{p\}}=\left(\mathbf{c}_{s}, \dot{\mathbf{c}}_{s}, \ddot{\mathbf{c}}_{s}\right)$ and $\mathbf{x}_{g}^{\{q\}}=\left(\mathbf{c}_{g}, \dot{\mathbf{c}}_{g}, \ddot{\mathbf{c}}_{g}\right)$ with $q \geq$ $p$, the transition feasibility problem consists in determining whether there exists a feasible trajectory $\mathbf{c}(t), t \in \mathbb{R}^{+}$of duration $T$, which connects exactly $\mathbf{x}_{s}^{\{p\}}$ and $\mathbf{x}_{g}^{\{q\}}$.

\section{B. Centroidal dynamic constraints on $\mathbf{c}(t)$}

For a contact phase $\{p\}$, for any $t \in[0, T]$ the centroidal dynamic constraints derived from the Newton-Euler equations are [4], [22]:

$$
\mathbf{H}^{\{p\}}\left[\begin{array}{c}
m(\ddot{\mathbf{c}}-\mathbf{g}) \\
m \mathbf{c} \times(\ddot{\mathbf{c}}-\mathbf{g})+\dot{\mathbf{L}}
\end{array}\right] \leq \mathbf{h}^{\{p\}}
$$

We isolate the state dependent term $\mathbf{w}$ :

$$
\underbrace{m \mathbf{H}^{\{p\}}}_{\mathbf{A}\{p\}} \underbrace{[(\mathbf{c} \times \ddot{\mathbf{c}}+\mathbf{g} \times \mathbf{c})+\dot{\mathbf{L}} / m]}_{\mathbf{w}} \leq \underbrace{\mathbf{h}^{\{p\}}+m \mathbf{H}^{\{p\}}\left[\begin{array}{l}
\mathbf{g} \\
\mathbf{0}
\end{array}\right]}_{\mathbf{a}\{p\}}
$$

where :

- $m \in \mathbb{R}$ is the mass of the robot;

- $\mathbf{g}=\left[\begin{array}{lll}0 & 0 & -9.81\end{array}\right]^{T}$ is the gravity vector;

- $\dot{\mathbf{L}} \in \mathbb{R}^{3}$ is the angular momentum (applied at $\mathbf{c}$ ).

- $\mathbf{H}^{\{p\}}$ and $\mathbf{h}^{\{p\}}$ are respectively a matrix and a vector defined by the contact points of the phase and their friction coefficients.

Because of the cross product between $\mathbf{c}$ and $\ddot{\mathbf{c}}$, the constraints are not linear, and the issue of finding a trajectory satisfying them in the general case is a non-convex problem.

\section{Centroidal kinematic constraints on $\mathbf{c}(t)$}

Each active contact creates kinematic constraints on $\mathbf{c}(t)$. We approximate them with linear constraints, depending on the $6 \mathrm{D}$ positions of each active contact frames, and refer the reader to [17] for the computation of these constraints, written $\mathbf{K}^{\{p\}} \mathbf{c} \leq \mathbf{k}^{\{p\}}$ for phase $\{\mathbf{p}\}$.

\section{Conservative ReFormulation OF THE TRANSITION PROBLEM}

To determine the existence of a valid $\mathbf{c}(t)$, we formulate the problem as a convex one by getting rid of the non-linear constraints induced by the cross product $\mathbf{c} \times \ddot{\mathbf{c}}$. To achieve this we impose a conservative condition on $\mathbf{c}(t)$. 


\section{A. Important note on the following equations.}

In what follows, we define several constant terms, which define the control points used in the various Bezier curves used by our method. Although in the end the obtained problem is solved straightforwardly, the manual calculation of these constants requires several simple mathematical operations and simplifications which we chose not to include in this paper for clarity and space reasons. At the $\mathrm{url}^{2}$, the reader can find a document detailing step by step the computation of such constants for the 0-step capturability problem. The methodology used in this document applies for all applications cases. At the $\mathrm{url}^{3}$ the reader will find a sympy script used to automatically compute the control points depending on the desired set of constraints. The control points only need to be computed once and for all.

\section{B. Lossless reformulation of $\mathbf{c}(t)$ as a Bezier curve}

Let us assume, without any loss of generality, that $\mathbf{c}(t)$ is an arbitrarily constrained Bezier curve of degree $n$ of unknown duration $T$ :

$$
\mathbf{c}(t)=\sum_{i=0}^{n} B_{i}^{n}(t / T) \mathbf{P}_{i}
$$

where the $B_{i}^{n}$ are the Bernstein polynomials and the $\mathbf{P}_{i}$ are control points.

To connect two states $\mathbf{x}_{s}^{\{p\}}$ and $\mathbf{x}_{g}^{\{q\}}$ with a Bezier curve it is necessary to take into account the positions, velocities and accelerations defined by the states. Accordingly, all the curves that we use comprise at least 6 control points, thus $n \geq 5$, to ensure that the following constraints are verified:

- $\mathbf{P}_{0}=\mathbf{c}_{s}$ and $\mathbf{P}_{n}=\mathbf{c}_{g}$ guarantee that the trajectory starts and ends at the desired locations;

- $\mathbf{P}_{1}=\frac{\dot{\mathbf{c}}_{s} / n}{T}+\mathbf{P}_{0}$ and $\mathbf{P}_{n-1}=\mathbf{P}_{n}-\frac{\dot{\mathbf{c}}_{g} / n}{T}$ guarantee that the trajectory initial and final velocities are respected;

- $\mathbf{P}_{2}=\frac{\ddot{\mathbf{c}}_{s} /(n(n-1))}{T^{2}}+2 \mathbf{P}_{1}-\mathbf{P}_{0}$ and $\mathbf{P}_{n-2}=\frac{\ddot{\mathbf{c}}_{g} /(n(n-1))}{T^{2}}+2 \mathbf{P}_{n-1}-\mathbf{P}_{n}$ guarantee that the initial and final accelerations are respected.

Depending on the considered problem, some or all of the constraints on the velocity and acceleration can be removed, without changing the validity of our approach. This genericity also allows to solve the 0 or 1-Step capturability problem, by removing the terminal position constraint. In the following we assume that the 6 constraints are present.

\section{Conservative reformulation of the transition problem}

We constrain $\mathbf{c}(t)$ to be a Bezier curve of degree $n=6$ and of duration $T$, which leaves a free control point $\mathbf{P}_{3}=\mathbf{y}$ :

$$
\mathbf{c}(t, \mathbf{y})=\sum_{i \in\{0,1,2,4,5,6\}} B_{i}^{6}(t / T) \mathbf{P}_{i}+B_{3}^{6}(t / T) \mathbf{y}
$$

In this case, $\mathbf{y}$ and $T$ are the only variables of the problem. For the time being, we fix $T$. We derive twice to obtain $\ddot{\mathbf{c}}(t)$,

\footnotetext{
${ }^{2}$ http://stevetonneau.fr/files/publications/ iros18/control_points.pdf

${ }^{3}$ http://stevetonneau.fr/files/publications/ iros18/derivate.py
}

and compute the cross product to get the expression of $\mathbf{w}(t)$. The non-convexity of the problem disappears, because the cross product of $\mathbf{y}$ by itself is $\mathbf{0}$, and all other terms are either constant or linear in $\mathbf{y}$. The non-convexity only disappears in our case, where the curve only admits one degree of freedom. $\mathbf{w}(t, \mathbf{y})$ is thus a six-dimensional Bezier curve of degree $2 n-$ 3 [23] (9 in this case) linearly dependent of $\mathbf{y}$ :

$$
\mathbf{w}(t, \mathbf{y})=\sum_{i \in\{0 . .9\}} B_{i}^{9}(t / T) \mathbf{w}_{i}(\mathbf{y})+\dot{\mathbf{L}}(t) / m
$$

where $\mathbf{w}_{i}(\mathbf{y}) \in \mathbb{R}^{6}$ are the control points expressed as :

$$
\mathbf{w}_{i}(\mathbf{y})=\mathbf{w}_{i}^{y} \mathbf{y}+\mathbf{w}_{i}^{s}
$$

The $\mathbf{w}_{i}^{y} \in \mathbb{R}^{6 \times 3}$ and $\mathbf{w}_{i}^{s} \in \mathbb{R}^{6}$ are constants that only depend on the control points $\mathbf{P}_{i}$ of $\mathbf{c}(t)$ and of $T$.

In what follows, for the sake of simplicity, we assume $\dot{\mathbf{L}}(t)=\mathbf{0}$. This is not a limitation: if we express $\dot{\mathbf{L}}(t)$ as a polynomial in the problem all the following reasoning stand. One way to include $\dot{\mathbf{L}}(t)$ is to represent it as a Bezier curve with one free variable, similarly to equation (4). However guaranteeing that we can generate a whole-body motion that tracks a variable $\dot{\mathbf{L}}(t)$ requires additional information on the whole-body motion, which we leave for future work.

The existence of a valid trajectory $\mathbf{c}(t)$ can thus be determined by solving a convex problem.

1) Application for a motion with no contact switch: Consider the case where $p=q=1$.

a) In the continuous case: Using the fact that a Bezier curve is comprised in the convex hull of its control points, and assuming that the start and goal states are feasible, we only need to find a $\mathbf{y}$ such that the $\mathbf{w}_{i}$ control points satisfy the constraints. This guarantees that the whole trajectory satisfies the constraints. We thus solve the feasibility problem with a minimal 3-dimensional Linear Program (LP) :

$$
\begin{array}{cl}
\text { find } & \mathbf{y} \\
\text { s.t. } & \mathbf{K}^{\{p\}} \mathbf{y} \leq \mathbf{k}^{\{p\}} \\
& \left(\mathbf{A}^{\{p\}} \mathbf{w}_{i}^{y}\right) \mathbf{y} \leq \mathbf{a}^{\{p\}}-\mathbf{A}^{\{p\}} \mathbf{w}_{i}^{s}, \forall i
\end{array}
$$

Constraining $\mathbf{y}$ to satisfy the constraints of the trajectory is a conservative approach that further constrains the solution space. However, this approach allows for a continuous solution to the problem.

b) In the discrete case: To remove the additional constraint on $\mathbf{y}$, we can proceed similarly to all existing approaches in the literature, and discretize the trajectory using a step $\Delta t$, which results in an increase in the constraint size. We discretize $\mathbf{w}(t, \mathbf{y})$ over $T$ as follows :

$$
\mathbf{w}(j \Delta t, \mathbf{y})=\mathbf{w}_{j}^{y} \mathbf{y}+\mathbf{w}_{j}^{s}
$$

Where $\mathbf{w}_{j}^{y}, \mathbf{w}_{j}^{s}$ are constants given by $\mathbf{P}_{\{0,1,2,4,5,6\}}$, the total duration $T$ and the time step $j \Delta t . j$ belongs to the phase set $J^{\{p\}}:\left\{j \in \mathbb{N}: 0 \leq j \Delta t \leq T^{\{p\}}\right\}$. Similarly we write $\mathbf{c}(j \Delta t, \mathbf{y})=\mathbf{c}_{j}^{y} \mathbf{y}+\mathbf{c}_{j}^{s}$. We can now rewrite inequality (2) expressed at the discretization point $j \Delta t$ :

$$
\left(\mathbf{A}^{\{p\}} \mathbf{w}_{j}^{y}\right) \mathbf{y} \leq \mathbf{a}^{\{p\}}-\mathbf{A}^{\{p\}} \mathbf{w}_{j}^{s}
$$


Thus we rewrite the LP (7) in a discretized form :

$$
\begin{array}{ll}
\text { find } & \mathbf{y} \\
\text { s.t. } & \underbrace{\left[\begin{array}{c}
\mathbf{K}^{\{p\}} \mathbf{c}_{j}^{y} \\
\mathbf{A}^{\{p\}} \mathbf{w}_{j}^{y}
\end{array}\right]}_{\mathbf{E}_{j}^{\{p\}}} \mathbf{y} \leq \underbrace{\left[\begin{array}{c}
\mathbf{k}^{\{p\}}-\mathbf{K}^{\{p\}} \mathbf{c}_{j}^{s} \\
\mathbf{a}^{\{p\}}-\mathbf{A}^{\{p\}} \mathbf{w}_{j}^{s}
\end{array}\right]}_{\mathbf{e}_{j}^{\{p\}}} \forall j \in J^{\{p\}}
\end{array} \forall
$$

2) Application for a motion with one contact switch: Consider the case where $q=p+1$. We define $T^{\{p\}}$ and $T^{\{q\}}$ the times spent in each phase, such that $T=T^{\{p\}}+T^{\{q\}}$. We also define $J^{\{q\}}:\left\{j \in \mathbb{N}, T^{\{p\}} \leq j \Delta t \leq T\right\}$.

We thus have 3 sets of constraints in this case: two for each phase, plus one for the transition time $t=T^{\{p\}}$ where the constraints of both phases apply:

$$
\begin{array}{ll}
\text { find } & \mathbf{y} \\
\text { s.t. } & \mathbf{E}_{j}^{\{z\}} \mathbf{y} \leq \mathbf{e}_{j}^{\{z\}} \quad, \forall j \in J^{\{z\}}, \forall z \in\{p, q\}
\end{array}
$$

3) Application for the general case: As we add more contact phases, we add additional set of constraints for each contact phase, and define as many $T^{\{z\}}$ and $J^{\{z\}}$ to write:

$$
\begin{array}{ll}
\text { find } & \mathbf{y} \\
\text { s.t. } & \mathbf{E}_{j}^{\{z\}} \mathbf{y} \leq \mathbf{e}_{j}^{\{z\}} \quad, \forall j \in J^{\{z\}}, \forall z \in\{p, \ldots, q\}
\end{array}
$$

In our experiments, we only consider three consecutive phases (which correspond to one step), and solve a new problem for each subsequent set of phases.

4) Cost function and additional constraint: With a small additional computational burden, we can formulate the problem (12) as a Quadratic Program (QP) and add a cost $l$. One can for instance minimize the integral of the squared acceleration norm or the angular momentum. This cost function is irrelevant to solve the transition feasibility problem, but it can be used to compute a warm start trajectory for a nonlinear solver (Section IV-D).

The formulation also allows to add arbitrary constraints on $\mathbf{c}$ and any of its derivatives by proceeding as done in equation (8). At the discretization point $j \Delta t$, we express the constraint as:

$$
\mathbf{O}_{j}^{\{z\}} \mathbf{y} \leq \mathbf{o}_{j}^{\{z\}}
$$

This allows to easily impose bounds on the velocity and acceleration of the center of mass or on the angular momentum variation. We obtain the generic QP :

$$
\begin{aligned}
\text { find } & \mathbf{y} \\
\text { minimize } & l(\mathbf{y}) \\
\text { s.t. } & \forall j \in J^{\{z\}}, \forall z \in\{p, \ldots, q\} \\
& \mathbf{E}_{j}^{\{z\}} \mathbf{y} \leq \mathbf{e}_{j}^{\{z\}} \\
& \mathbf{O}_{j}^{\{z\}} \mathbf{y} \leq \mathbf{o}_{j}^{\{z\}}
\end{aligned}
$$

In our experiments we set constraints on the acceleration and velocity and minimize the squared acceleration norm.

\section{APPliCATIONS TO THE CONTACT PLANNING PROBLEM AND THE COMPUTATION OF A WARM START TRAJECTORY}

We first study the application of CROC for contact planning. We recall that the goal of contact planning is to provide a sequence of contact phases to a motion generation method.

\section{A. CROC as a transition feasibility criterion}

We use our contact planner [15] to demonstrate the interest of CROC as a feasibility criterion. Given a state $\mathbf{x}_{s}^{\{p\}}=\left(\mathbf{c}_{s}, \dot{\mathbf{c}}_{s}, \ddot{\mathbf{c}}_{s}\right)$ and a trajectory for the root of the robot computed by [16], the planner generates either a candidate state $\mathbf{x}_{g}^{\{p+1\}}$ or $\mathbf{x}_{g}^{\{p+2\}}$, such that $\mathbf{x}_{g}^{\{z\}}=\left(\mathbf{c}_{g}, \dot{\mathbf{c}}_{g}, \ddot{\mathbf{c}}_{g}\right)$ with $z$ either $p+1$ or $p+2$. We use CROC to determine whether there exists a trajectory that connects exactly both states, in which case we keep the candidate state, otherwise we request the contact generator for a new candidate.

Figure 2 shows examples of unfeasible contact sequences: while all the states in these sequences are dynamically consistent, there is no feasible transitioning motion between them. These invalid sequences are filtered out by CROC, resulting in a more robust planner.

\section{B. Time sampling}

To remain convex, we choose not to include the duration of each phase $T^{\{p\}}, T^{\{p+1\}}$ and $T^{\{p+2\}}$ as variables of CROC. We rather sample various combinations of times and solve the corresponding QPs in sequence until a solution is found. In theory, this would mean that we need to sample an infinity of combinations in order to be complete. However, we pragmatically reduce this number and give up on the completeness while maintaining a high success rate as follows. We sampled a time for each duration phase $T^{\{z\}}$ by choosing a value between 0.2 and 2 seconds, with increments of $50 \mathrm{~ms}$. For a sequence of three phases, this gives a total of 46656 possible combinations. We tested CROC with all these combinations on various problems : with HRP2 or HyQ robots on flat and non-coplanar surfaces, for several thousands of states.

Upon analysis of the results of the convergence of the QPs, we found out that we can use a small list of timings combinations ( 5 in our case) that covers $100 \%$ of the success cases for all the robots and scenarios tested. We thus solve a maximum of 5 QPs for each validation.

\section{Experimental results}

1) Computation time: Solving the QP (14) for three consecutive contact phases takes on average 40 microseconds ${ }^{4}$. Computing the whole feasibility test (with the small list of timings as described above) takes on average $2.9 \mathrm{~ms}, 85 \%$ of it is spent computing the equilibrium constraints $\mathbf{H}$ and $\mathbf{h}^{5}$.

While we are not solving the complete centroidal dynamic optimization problem as in [24], [9] (we are only interested in the existence of at least one solution and we over-constrain

\footnotetext{
${ }^{4}$ Implemented in c++ with QuadProg, measured with a computer with an Intel Xeon CPU E5-1630 v3 at $3.70 \mathrm{GHz}$

${ }^{5}$ Computed with [22]
} 


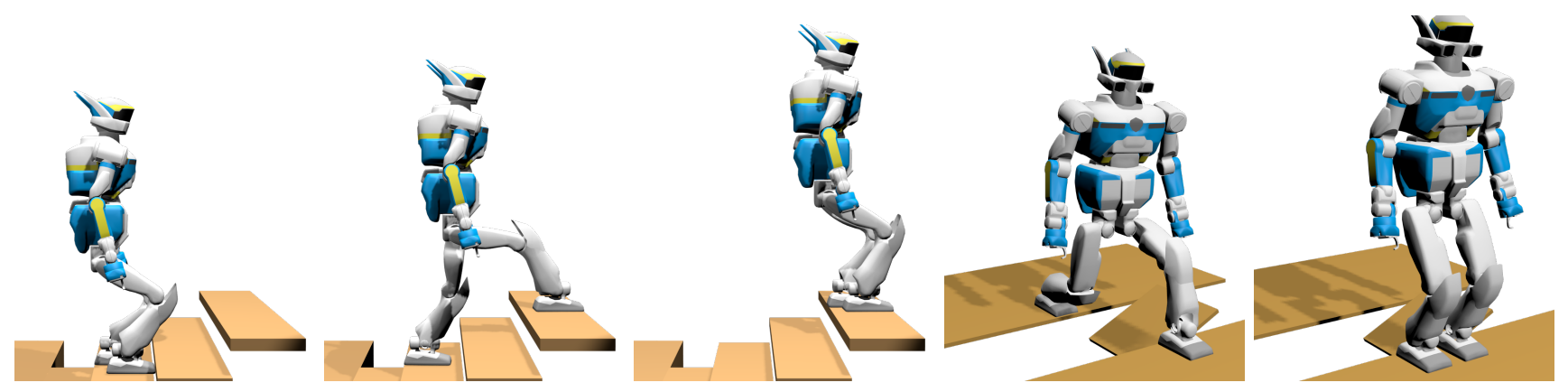

Fig. 2: Examples of unfeasible contacts transitions found and rejected by CROC.
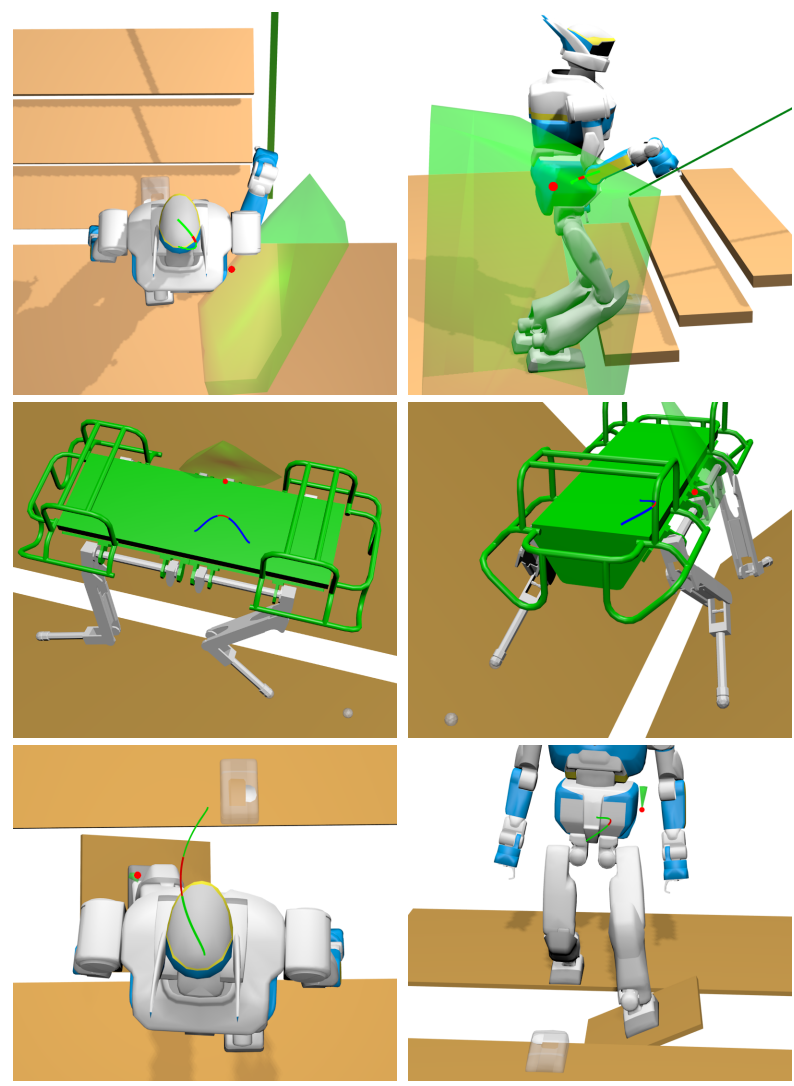

Fig. 3: Examples of trajectories found by our method. Green polytopes : valid position of $\mathbf{y}$ that verifies the constraints of the problem (14), red sphere : solution found for $\mathbf{y}$. The red part of the trajectory is for the phase with $n_{c}-1$ active contacts. The next contact is shown in transparency.

$\mathbf{c}(t)$ ), it can be noted that our method performs at least one order of magnitude faster. This allows us to test many candidate states for feasibility during the contact planning phase, and still achieve interactive performances.

2) Contact planning: We tested our contact planner with the robots HyQ and HRP2 on various scenarios. Several contact sequences and whole body motions are shown in the attached video. We did not observe significant improvements for simple scenarios (such as walks on flat ground), because nearly all the transitions found by our previous heuristic were feasible. However, we observed a significant change in the contacts plans computed for harder, multi-contact, scenarios. Our feasibility criterion filters out all the unfeasible transitions such as the ones depicted in Figure 2, for which CROC is unfeasible.

Figure 3 shows sample trajectories found with our method for feasible transitions, along with the valid region for $\mathbf{y}$ and the position of $\mathbf{y}$ found with our cost function. On the top row, HRP2 is climbing a stair using his right hand. In the middle row, HyQ is walking between two $45^{\circ}$ inclined planes.

On the last row and on figure 4 HRP2 crosses a gap which is too large to be crossed with only one step (while the transition is kinematically feasible, no dynamically consistent whole body motion was found for this long step using either state of the art solver). The platform in the middle of the gap is inclined such that there is no way to remain in static equilibrium while solely resting on it. In other words, this scenario cannot be solved with a quasi-static motion. Without CROC, the planner will produce contacts sequences that randomly try to cross the gap in one step or use the platform with the wrong foot, resulting in unfeasible problems.

With CROC, the only contact plans computed use a contact between the left foot and the platform, as shown in Figure 4 , resulting in the feasible whole body motion shown in the companion video.

\section{Motion generation}

In order to generate a whole body motion from our contact sequence, we used the framework proposed in [24]. We automatically compute a contact sequence with our contact planner and CROC. Their non-linear solver then computes an optimal centroidal trajectory, using a multiple-shooting algorithm. Finally, a second-order inverse kinematics solver computes a whole body motion that follows the computed trajectory. The whole body motion of the sequence of contacts in Figure 4 is shown in the attached video.

Because trajectory generation methods use non-linear solvers, the choice of an initial guess is essential as it can drastically change the result of the method. Choosing this initial guess may be challenging for multi-contact motions. The centroidal trajectory $(\mathbf{c}(t), \dot{\mathbf{c}}(t), \ddot{\mathbf{c}}(t))$ found by CROC can be used as an initial guess to warm start the non-linear 


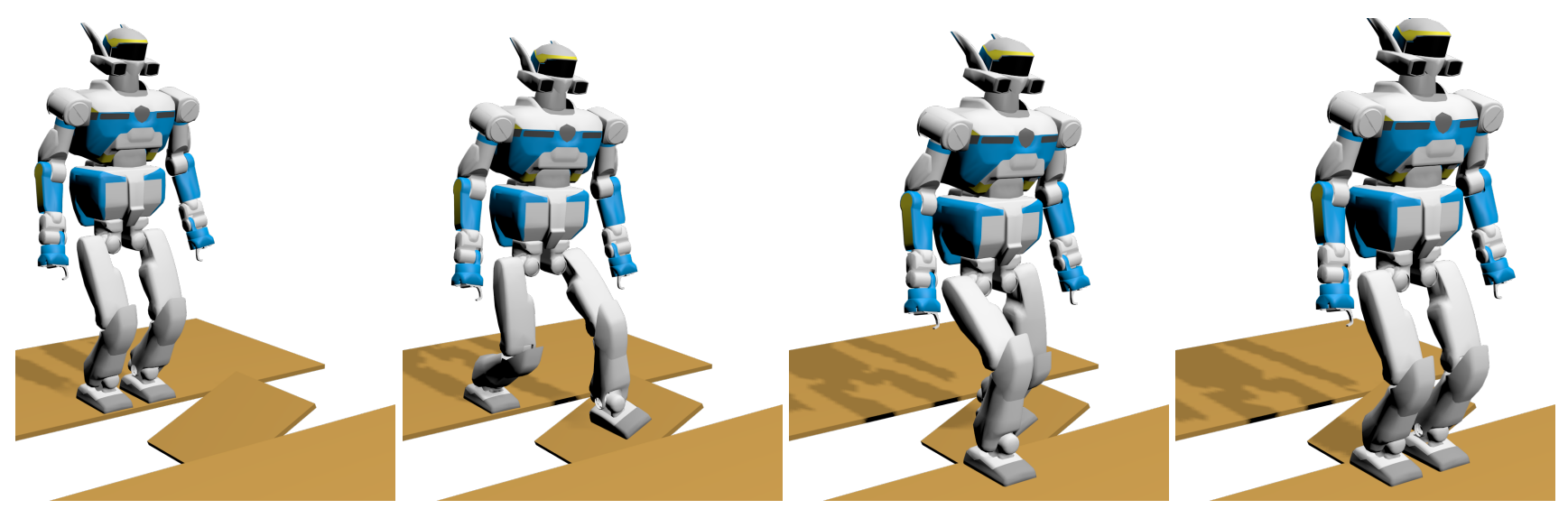

Fig. 4: Contact plan found by our planner. The platform is inclined at $30^{\circ}$, such that no states can be in static equilibrium on the platform.

solvers. We tested the method presented in [24] with a CROC warm start or with their naive one, on hundreds of randomly generated scenarios. In our tests, we observed that in $17 \%$ of the cases, the solver only converged with our warm-start. In the other cases, it converged with both initial guesses but we noticed a decrease of an average of $21 \%$ of the time required to converge when we used our warm start.

\section{CONCLUSION AND DISCUSSION}

In this paper we introduce an accurate and efficient formulation of the centroidal dynamics of a legged robot, which is convex and not restricted to quasi-static motions. To our knowledge it is the first method to combine these three properties. We demonstrate the interest of the method as a transition feasibility criterion for the contact planning problem, and for computing a warm start centroidal trajectory for non-linear solvers.

Contrary to methods using simplifying assumptions or approximations, one strong advantage is that the computed trajectories are thus guaranteed to respect the centroidal dynamics of the system. The additional main advantage of the approach lies in its computational efficiency, which makes it the first method fast enough to be integrated within a contact planner. The last one is its simplicity of implementation and the fact that it does not require any form of parametrization.

\section{A. Handling whole-body approximations and uncertainties}

The remaining source of approximation is shared with all centroidal-based methods, and comes from the whole-body constraints (joint limits, angular momentum and torques), which are only approximated or ignored in the current formulation. One solution could be to alternate centroidal optimization with whole-body optimization as other approaches do [9], however for the transition feasibility problem, this approach would result in an increased computational burden that is not compatible with the combinatorial aspect of the search. One way to improve the quality of this approximation is to integrate torque constraints [25], [26]. Expressing such constraints at the COM level is considered for future work.
Another interesting question is to guarantee that CROC provides an answer robust to real world uncertainties. One option to address the issue is to add an additional slack variable to the problem in order to maximize the distance to the considered constraints, similarly to our previous work [15], and reject solutions that would not reach a user-defined threshold. This would result in a conservative yet robust approach to guarantee the transition.

\section{B. How conservative is CROC?}

The price for convexity is that our method does not cover the whole solution space. However, evaluating the actual loss is not possible since we share the limitation with all the existing approaches, and thus do not have a ground truth for comparison. For future work we plan to compare our method with a non-linear solver [8], which we will consider as the ground truth to measure the effective loss.

Comparatively to this non-linear solver, with the same cost function CROC will most likely find a less optimal trajectory in general. This is not a problem because we are mostly concerned with feasibility. Regarding the time variable, it appears that sampling the time over a discretized set has no significant impact on the success rate on the method, and thus is not an issue regarding the feasibility problem.

Interestingly, our experiments suggest that the solution set spanned by CROC is not strictly included in the one spanned by a non-linear solver not warm-started with CROC. CROC is able to help non-linear solvers to converge in cases where they fail otherwise. We also observe that our warm starts significantly improve the computational performance of the non-linear solver. This demonstrates another quantitative interest of the approach, but further analysis is required to provide a better insight on the benefit of combining the approaches for motion generation.

\section{Application to 0 and 1 step capturability}

The N-Step capturability problem consists in determining the ability of a robot (in a given state) to come to a stop (ie. null velocity and acceleration) without falling by taking at most $\mathrm{N}$ steps. It is used to detect and prevent fall. 
We can easily change the constraints on $\mathbf{c}(t)$ defined in subsection III-B to remove the constraint on $\mathbf{c}_{g}$ and constrain $\left(\dot{\mathbf{c}}_{g}=\mathbf{0}, \ddot{\mathbf{c}}_{g}=\mathbf{0}\right)$. With this set of constraints, the feasibility of LP (7) determines the 0-Step capturability. Similarly, LP (11) determines the 1-Step capturability.

For future work we would like to empirically determine the accuracy of our method with respect to this problem, using a framework similar to [20].

\section{SOURCE CODE}

Code available (C++/python) under a BSD-2 license: https://gitlab.com/stonneau/bezier_CoM_traj

\section{ACKNOWLEDGEMENTS}

Supports: ANR LOCO3D ANR-16-CE33-0003, ERC Actanthrope ERC-2013-ADG, H2020 Memmo ICT-780684.

\section{REFERENCES}

[1] S. Kajita, F. Kanehiro, K. Kaneko, K. Fujiwara, K. Harada, K. Yokoi, and H. Hirukawa, "Biped Walking Pattern Generation by using Preview Control of Zero-Moment Point," in Proc. of IEEE Int. Conf. Robot. and Auto (ICRA), Taipei, Taiwan, Sept. 2003.

[2] A. Escande, A. Kheddar, and S. Miossec, "Planning contact points for humanoid robots," Robotics and Autonomous Systems, vol. 61, no. 5, pp. 428 - 442, 2013. [Online]. Available: http://www.sciencedirect.com/science/article/pii/S0921889013000213

[3] J. Pratt, J. Carff, S. Drakunov, and A. Goswami, "Capture Point: A Step toward Humanoid Push Recovery," 2006 6th IEEE-RAS International Conference on Humanoid Robots, 2006.

[4] Z. Qiu, A. Escande, A. Micaelli, and T. Robert, "Human motions analysis and simulation based on a general criterion of stability," in Int. Symposium on Digital Human Modeling, 2011.

[5] M. X. Grey, A. D. Ames, and C. K. Liu, "Footstep and motion planning in semi-unstructured environments using randomized possibility graphs," 2017.

[6] T. Bretl, S. Rock, J.-C. Latombe, B. Kennedy, and H. Aghazarian, "Free-climbing with a multi-use robot." in ISER, ser. Springer Tracts in Advanced Robot., M. H. A. Jr. and O. Khatib, Eds., vol. 21. Springer, 2004, pp. 449-458.

[7] K. Hauser, T. Bretl, and J.-C. Latombe, "Non-gaited humanoid locomotion planning," in Humanoid Robots, 2005 5th IEEE-RAS Int. Conf. on, 2005, pp. 7-12.

[8] J. Carpentier and N. Mansard, "Multi-contact locomotion of legged robots," Rapport LAAS n 17172. https://hal.laas.fr/hal-01520248. Conditionally accepted for IEEE Trans. on Robotics, 2017.

[9] A. Herzog, N. Rotella, S. Schaal, and L. Righetti, "Trajectory generation for multi-contact momentum-control," in Humanoid Robots (Humanoids), 15h IEEE-RAS Int. Conf. on, Nov. 2015.

[10] H. Dai, A. Valenzuela, and R. Tedrake, "Whole-body motion planning with centroidal dynamics and full kinematics," in Humanoid Robots (Humanoids), 14th IEEE-RAS Int. Conf. on, Madrid, Spain, 2014, pp. 295-302.

[11] S. Caron, A. Escande, L. Lanari, and B. Mallein, "Capturabilitybased analysis, optimization and control of $3 \mathrm{~d}$ bipedal walking," Jan. 2018, submitted. [Online]. Available: https://hal.archives-ouvertes.fr/ hal-01689331
[12] R. Deits and R. Tedrake, "Footstep planning on uneven terrain with mixed-integer convex optimization," in Humanoid Robots (Humanoids), 14th IEEE-RAS Int. Conf. on, Madrid, Spain, 2014.

[13] B. Ponton, A. Herzog, S. Schaal, and L. Righetti, "A convex model of humanoid momentum dynamics for multi-contact motion generation," in Proceedings of the 2016 IEEE-RAS International Conference on Humanoid Robots, 2016.

[14] I. Mordatch, E. Todorov, and Z. Popović, "Discovery of complex behaviors through contact-invariant optimization," ACM Trans. on Graph., vol. 31, no. 4, pp. 43:1-43:8, 2012.

[15] S. Tonneau, A. D. Prete, J. Pettr, C. Park, D. Manocha, and N. Mansard, "An efficient acyclic contact planner for multiped robots," IEEE Transactions on Robotics, vol. 34, no. 3, pp. 586-601, June 2018.

[16] P. Fernbach, S. Tonneau, A. D. Prete, and M. Taïx, "A kinodynamic steering-method for legged multi-contact locomotion," in 2017 IEEE/RSJ International Conference on Intelligent Robots and Systems (IROS), Sept 2017, pp. 3701-3707.

[17] S. Tonneau, P. Fernbach, A. D. Prete, J. Pettré, and N. Mansard, "2PAC: Two Point Attractors for Center of Mass Trajectories in Multi Contact Scenarios," July 2018, to appear in Trans. on Graphics. [Online]. Available: https://hal.archives-ouvertes.fr/hal-01609055

[18] T. Koolen, T. de Boer, J. R. Rebula, A. Goswami, and J. E. Pratt, "Capturability-based analysis and control of legged locomotion, part 1: Theory and application to three simple gait models," I. J. Robotics Res., vol. 31, no. 9, pp. 1094-1113, 2012. [Online]. Available: https://doi.org/10.1177/0278364912452673

[19] J. E. Pratt, T. Koolen, T. de Boer, J. R. Rebula, S. Cotton, J. Carff, M. Johnson, and P. D. Neuhaus, "Capturability-based analysis and control of legged locomotion, part 2: Application to $\mathrm{m} 2 \mathrm{v} 2$, a lowerbody humanoid," I. J. Robotics Res., vol. 31, no. 10, pp. 1117-1133, 2012. [Online]. Available: https://doi.org/10.1177/0278364912452762

[20] A. Del Prete, S. Tonneau, and N. Mansard, "Zero Step Capturability for Legged Robots in Multi Contact," Accepted on IEEE Trans on Robotics, 2018. [Online]. Available: https://hal.archives-ouvertes.fr/ hal-01574687

[21] D. E. Orin, A. Goswami, and S.-H. Lee, "Centroidal dynamics of a humanoid robot," Autonomous Robots, vol. 35, no. 2, pp. 161-176, Oct 2013.

[22] A. Del Prete, S. Tonneau, and N. Mansard, "Fast Algorithms to Test Robust Static Equilibrium for Legged Robots," in Proc. of IEEE Int. Conf. on Robot. and Auto (ICRA), Stockholm, Sweden, 2016.

[23] J. M. (https://math.stackexchange.com/users/305862/jean marie), "Is the cross product of two bezier curves a bezier curve?" Mathematics Stack Exchange, uRL:https://math.stackexchange.com/q/2228976 (version: 2017-12-10). [Online]. Available: https://math. stackexchange.com/q/2228976

[24] J. Carpentier, R. Budhiraja, and N. Mansard, "Learning Feasibility Constraints for Multi-contact Locomotion of Legged Robots," in Robotics: Science and Systems, Cambridge, MA, United States, July 2017. [Online]. Available: https://hal.laas.fr/hal-01526200

[25] R. Orsolino, M. Focchi, C. Mastalli, H. Dai, D. G. Caldwell, and C. Semini, "Application of wrench based feasibility analysis to the online trajectory optimization of legged robots," IEEE Robotics and Automation Letters, pp. 1-1, 2018.

[26] V. Samy, S. Caron, K. Bouyarmane, and A. Kheddar, "Post-impact adaptive compliance for humanoid falls using predictive control of a reduced model," in 2017 IEEE-RAS 17th International Conference on Humanoid Robotics (Humanoids), Nov 2017, pp. 655-660. 